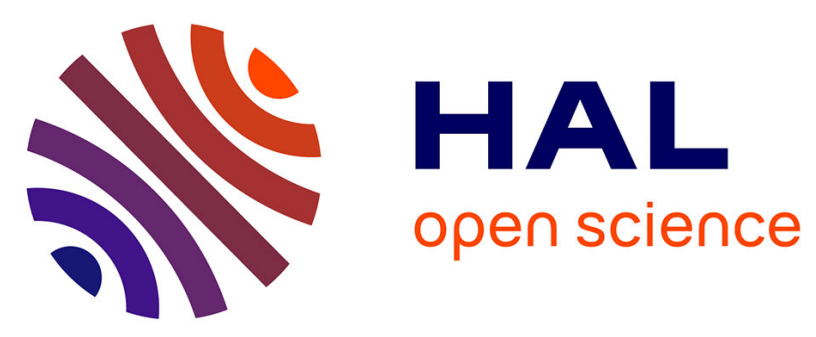

\title{
A methodology for generating normal and pathological brain perfusion SPECT images for evaluation of MRI/SPECT fusion methods: application in epilepsy.
}

Christophe Grova, Pierre Jannin, Arnaud Biraben, I. Buvat, Habib Benali, Anne-Marie Bernard, Jean Marie Scarabin, Bernard Gibaud

\section{To cite this version:}

Christophe Grova, Pierre Jannin, Arnaud Biraben, I. Buvat, Habib Benali, et al.. A methodology for generating normal and pathological brain perfusion SPECT images for evaluation of MRI/SPECT fusion methods: application in epilepsy.. Physics in Medicine and Biology, 2003, 48 (24), pp.4023-43. inserm-00332761

\section{HAL Id: inserm-00332761 https://www.hal.inserm.fr/inserm-00332761}

Submitted on 21 Oct 2008

HAL is a multi-disciplinary open access archive for the deposit and dissemination of scientific research documents, whether they are published or not. The documents may come from teaching and research institutions in France or abroad, or from public or private research centers.
L'archive ouverte pluridisciplinaire HAL, est destinée au dépôt et à la diffusion de documents scientifiques de niveau recherche, publiés ou non, émanant des établissements d'enseignement et de recherche français ou étrangers, des laboratoires publics ou privés. 


\section{A methodology for generating normal and pathological brain perfusion SPECT images for evaluation of MRI/SPECT fusion methods: application in epilepsy}

C. Grova $\dagger \| \S$, P. Jannin†, A. Biraben $\dagger$, I. Buvat $\ddagger$ H. Benali A.M. Bernard\#, J.M. Scarabin $\dagger$ and B. Gibaud $\dagger$

† Laboratoire IDM, Faculté de Médecine, Université de Rennes 1, Rennes, France

|| Montreal Neurological Institute, McGill University, Montreal, Canada

$\ddagger$ INSERM U494, CHU Pitié Salpêtrière, Paris, France

\# Service de Médecine Nucléaire, Centre Eugène Marquis, Rennes, France

$\S$ To whom correspondence should be addressed (christophe.grova@mail.mcgill.ca) 
Abstract. Quantitative evaluation of brain MRI/SPECT fusion methods for normal and in particular pathological data sets is difficult, due to the frequent lack of relevant ground truth. We propose a methodology to generate MRI and SPECT data sets dedicated to the evaluation of MRI/SPECT fusion methods and illustrate the method when dealing with ictal SPECT.

The method consists in generating normal or pathological SPECT data perfectly aligned with a high-resolution 3D T1-weighted MRI using realistic Monte Carlo simulations that closely reproduce the response of a SPECT imaging system. Anatomical input data for the SPECT simulations are obtained from this 3D T1weighted MRI, while functional input data result from an inter-individual analysis of anatomically standardised SPECT data. The method makes it possible to control the "brain perfusion" function by proposing a theoretical model of brain perfusion from measurements performed on real SPECT images. Our method provides an absolute gold standard for assessing MRI/SPECT registration method accuracy since, by construction, the SPECT data are perfectly registered with the MRI data.

The proposed methodology has been applied to create a theoretical model of normal brain perfusion and ictal brain perfusion characteristic of mesial temporal lobe epilepsy. To approach realistic and unbiased perfusion models, real SPECT data were corrected for uniform attenuation, scatter and partial volume effect. An anatomic standardisation was used to account for anatomic variability between subjects. Realistic simulations of normal and ictal SPECT deduced from these perfusion models are presented. The comparison of real and simulated SPECT images showed relative differences in regional activity concentration less than $20 \%$ in most anatomical structures, for both normal and ictal data, suggesting realistic models of perfusion distributions for evaluation purposes. Inter-hemispheric asymmetry coefficients measured on simulated data were found within the range of asymmetry coefficients measured on corresponding real data.

The features of the proposed approach are compared with those of other methods previously described to obtain data sets appropriate for the assessment of fusion methods.

\section{Introduction}

Single photon emission computed tomography (SPECT) using ${ }^{99 m} \mathrm{Tc}-\mathrm{HMPAO}$ or ${ }^{99 m} \mathrm{Tc}-$ ECD as a radiotracer is the most widely applied technique to study cerebral blood flow [Patterson and Wyper 1997]. It is used in patients with various diseases of the central nervous system including epilepsy [Ho et al 1996, Devous et al 1998]. In patients with epilepsy, ictal images are obtained by injecting these radiotracers at the onset of the seizure and acquiring SPECT data within two hours after injection. Ictal SPECT provides considerable information regarding the epileptogenic focus (97\% sensitivity in temporal lobe epilepsy [Devous et al 1998]) and is currently the only imaging technique available to explore the ictal state.

MRI/SPECT image registration and fusion methods are extremely useful to overcome the problems of the imprecise anatomical landmarks and low spatial resolution of SPECT images. These methods also facilitate a more objective detection and localisation of perfusion abnormalities. Since only relative quantification of perfusion is 
available from ${ }^{99 m}$ Tc-HMPAO or ${ }^{99 m}$ Tc-ECD SPECT data [Patterson and Wyper 1997], image analysis is usually performed by comparing the two brain hemispheres (e.g., [Ho et al 1996, Lobaugh et al 2000, Aubert-Broche et al 2003]), by comparing different states in the same patient (for example subtraction of the ictal data from inter-ictal data in patients with epilepsy [Zubal et al 1995, O'Brien et al 1998]), or by comparing patient groups (e.g., diseased patients versus healthy controls [Acton and Friston 1998, Lee et al 2000]). These kind of image comparisons may also help study the impact of different parameters on brain perfusion: e.g., the effect of the imaging device, the effect of gender and age of the studied population (see [Van Laere et al 2001] for a detailed analysis of the perfusion of 89 healthy subjects). The clinical evaluation of MRI/SPECT brain image fusion methods is difficult, especially for pathological cases, due to the frequent lack of relevant ground truth.

Digital simulations of imaging modalities help the better understanding of the behaviour of SPECT/MRI registration methods [Thurjfell et al 2000]. Realistic simulations are usually obtained from high-resolution human brain data, defining a spatial model of brain anatomy [Zubal et al 1994, Collins et al 1998]. After generating high-resolution activity maps on such a spatial model, realistic simulations such as Monte Carlo simulations in nuclear medicine [Zaidi 1999] closely model the physical phenomena involved in the generation and acquisition of the SPECT data.

We propose here a methodology for performing SPECT simulations from an anatomical support given by a high-resolution T1-weighted 3D MRI. Functional input data for the SPECT simulations were obtained from an inter-individual analysis of SPECT data, using anatomical standardisation techniques. In order to control the "brain perfusion" function, realistic normal and pathological brain perfusion models were obtained from the analysis of anatomically standardised SPECT data. After defining realistic activity distributions from these group analyses, Monte Carlo simulations were performed to generate realistic SPECT data for which the ground truth was known. Our method thus produces an absolute gold standard, suitable to evaluate the matching of the SPECT and MRI data sets. This generic model was applied to generate normal SPECT data and characteristic ictal SPECT data observed in mesial temporal lobe epilepsy (MTLE). Volume of Interest (VOI)-based perfusion measurements performed on simulated data were compared to similar measurements performed on real data to check for the relevance of SPECT simulations.

\section{Material and methods}

Monte Carlo simulations of SPECT data require the definition of an activity map representing the $3 \mathrm{D}$ spatial distribution of the radiotracer $\left({ }^{99 m} \mathrm{Tc}-\mathrm{HMPAO}\right.$ or ${ }^{99 m} \mathrm{Tc}-$ ECD) and of the associated attenuation map describing the attenuation properties of the body. The definition of these maps requires a spatial model of brain anatomy and a theoretical model of brain perfusion, to assign attenuation and activity values to each anatomical structure. 


\subsection{Spatial model of brain anatomy}

The spatial model of brain anatomy used in this study was deduced from the anthropomorphic model of the head proposed by [Zubal et al 1994]. This original model was established from a high-resolution 3D T1-weighted MRI of a healthy subject (124 axial slices, matrix: $256 \times 256$, voxel size: $\left.1.1 \times 1.1 \times 1.4 \mathrm{~mm}^{3}\right)$ from which sixty-three intra- and extra-cerebral anatomical structures, or VOIs, were hand drawn and labelled.

To simulate SPECT scans obtained in patients with epilepsy, we segmented some intra-cerebral VOIs of the Zubal phantom and we distinguished bilateral VOIs. Characterisation of VOIs for each hemisphere was needed as most brain perfusion studies in epilepsy compare the healthy hemisphere with the diseased hemisphere [Ho et al 1996, Devous et al 1998]. Further segmentation of the Zubal phantom was required as the anatomical regions that are usually considered by epilepsy specialists divide the lobes into different cortical zones (e.g., [Bartolomei et al 1999]). These segmentations were performed semi-automatically by an experienced operator who located the proportional grid proposed by the Talairach and Tournoux atlas [Talairach and Tournoux 1988] on the MRI of the Zubal phantom. The operator segmented the Zubal phantom VOIs corresponding to the temporal, frontal and occipital lobes, by identifying the corresponding anatomical sub-structures on the Talairach and Tournoux atlas. The resulting cerebral VOIs are listed in Table 1. Lateralised VOIs were then obtained by interactively defining the inter-hemispheric plane. They define the cerebral entities of our spatial model of brain anatomy. These $N_{a}=2 \times 20=40$ intra-cerebral lateralised VOIs were used for the anatomical standardisation analysis of real SPECT data from which theoretical models of perfusion were derived (see $\S 2.3$ ).

\subsection{Photon attenuation map}

A theoretical map of photon attenuation coefficients was defined at the resolution of the spatial model described in $\S 2.1$. This map was obtained by assigning a tissue type together with the associated attenuation coefficient $\mu$ at the $140 \mathrm{keV}$ energy emission of ${ }^{99 m} \mathrm{Tc}$ to each VOI. Seven tissue types were considered: connective tissue $\left(\mu=0.1781 \mathrm{~cm}^{-1}\right)$, water $\left(\mu=0.1508 \mathrm{~cm}^{-1}\right)$, brain $\left(\mu=0.1551 \mathrm{~cm}^{-1}\right)$, bone $\left(\mu=0.3222 \mathrm{~cm}^{-1}\right)$, muscle $\left(\mu=0.1553 \mathrm{~cm}^{-1}\right)$, fat $\left(\mu=0.1394 \mathrm{~cm}^{-1}\right)$, and blood $\left(\mu=0.1585 \mathrm{~cm}^{-1}\right)$.

\subsection{Activity map: theoretical model of brain perfusion}

To define the activity map, a theoretical model of brain perfusion was established from real SPECT data using an anatomical standardisation method (see Figure 1). Similar approaches using anatomically standardised VOIs to perform measurements were previously described, e.g., [Koyama et al 1997, Lobaugh et al 2000, Van Laere et al 2001, Kang et al 2001, Pagani et al 2002]. Our approach consisted in a spatial transformation of the SPECT data to a mean anatomical reference volume (described in §2.3.3) fol- 
lowed by quantitative measurements in the VOIs of the spatial model of brain anatomy, previously registered to the same reference volume. This generic method was applied here to obtain a normal perfusion model and a characteristic ictal perfusion model for MTLE.

2.3.1. Population of subjects: Two populations of subjects exhibiting characteristic perfusion patterns were considered. SPECT scans of 27 healthy subjects (12 men and 15 women, aged 20 to 56 years, mean: 37.6 years) provided by Dr. L. Barnden Ph.D. (Queen Elizabeth Hospital, Woodville, Australia) were used to derive a theoretical model of normal perfusion. Ten MTLE patients ( 6 men and 4 women, aged 19 to 43 years, mean: 33.5 years) from the epileptology and neurosurgery units of the Rennes university hospital (Rennes, France) were selected to derive a model of ictal perfusion. After clinical investigations including SPECT acquisitions, they all underwent curative surgery and are now seizure free. SPECT data were acquired after perictal injections of the radiotracer at seizure onset, detected using video-electroencephalography monitoring. The diagnosis of MTLE with a mesial temporal focus and lateral temporal propagation was established on the basis of electroclinical data in accordance with the classification of temporal lobe epilepsy proposed by [Bartolomei et al 1999]. The surgical outcome, after at least two years from the surgery confirmed the diagnosis.

2.3.2. Data acquisition and pre-processing: For the 27 healthy subjects, SPECT images were acquired with a three-head gamma camera equipped with ultra highresolution parallel collimators (IRIX, Philips Medical Systems) after injection of 500 $\mathrm{MBq}$ of ${ }^{99 m} \mathrm{Tc}-\mathrm{HMPAO}$. One hundred and twenty projections over $360^{\circ}$ (matrix: $128 \times 128$, pixel size: $3.59 \mathrm{~mm}$ ) were acquired using a $20 \%$ energy window centred on $140 \mathrm{keV}(126-154 \mathrm{keV})$ and a $(111-125 \mathrm{keV})$ Compton window simultaneously. The Jaszczak method [Jaszczak et al 1985] was used for scatter correction by subtracting the projections corresponding to the Compton window, multiplied by $k=1.3$, from the $20 \%$ projections. This weighting factor has been found appropriate for the considered Compton window [Barnden et al 2002].

For the ten MTLE patients, the ictal SPECT images were acquired with a twohead DST-XL camera (General Electric Medical Systems) equipped with ultra highresolution fan beam collimators (7 patients) or ultra high-resolution parallel collimators (3 patients), after injecting $740 \mathrm{MBq}$ of ${ }^{99 m} \mathrm{Tc}-\mathrm{HMPAO}$ or ${ }^{99 m} \mathrm{Tc}-\mathrm{ECD}$ depending on availability. Sixty-four projections over $360^{\circ}$ (matrix: $128 \times 128$, pixel size: $4.51 \mathrm{~mm}$ ) were acquired using a 20\% energy window centred on $140 \mathrm{keV}(126-154 \mathrm{keV})$. No simultaneous acquisition in a Compton window was available for these retrospective data. 3D T1-weighted MRI was also performed in these ten patients, using a $1.5 \mathrm{~T}$ Signa machine (General Electric Medical Systems) with the following characteristics: 124 sagittal $256 \times 256$ slices, voxel size: $0.9375 \times 0.9375 \times 1.3 \mathrm{~mm}^{3}$, SPGR sequence (FOV: $24 \mathrm{~cm}, \alpha: 30^{\circ}$, TE: $3 \mathrm{~ms}$, TR: $33 \mathrm{~ms}$ ).

SPECT data were reconstructed using filtered backprojection with a ramp filter 
(Nyquist frequency cutoff). The reconstructed data were post-filtered with an $8 \mathrm{~mm}$ full width at half maximum ( $F W H M)$ 3D Gaussian filter. The acquisition of linear sources using the clinical acquisition and reconstruction protocols yielded, in the reconstructed images, a spatial resolution of $F W H M=12.2 \mathrm{~mm}$ both for the DST-XL tomograph (Rennes) and for the IRIX tomograph (Woodville). Assuming uniform attenuation in the head, first order Chang attenuation correction [Chang 1978] was performed, with the attenuation coefficient $\mu=0.15 \mathrm{~cm}^{-1}$ for scatter corrected data and $\mu=0.12 \mathrm{~cm}^{-1}$ for uncorrected data [Jaszczak et al 1985]. Relative quantification errors when assuming uniform attenuation instead of non-uniform attenuation were shown to be lower than $5 \%$ in ${ }^{123}$ I-IMP perfusion SPECT studies [Iida et al 1998], when the head mask was properly defined on the SPECT data. Thus, we assumed uniform attenuation correction to be sufficiently accurate for our purpose. For healthy subjects, the head mask was obtained by thresholding the SPECT volumes reconstructed from the Compton window data, as recommended when SPECT transmission acquisition is not available. For each MTLE patient, the head mask was obtained by background removal of the MRI data, each head mask was then matched to the corresponding SPECT data after MRI/SPECT registration (described in $\$ 2.3 .3$ ). In the following, attenuation corrected SPECT data will be referred to as AC SPECT data, whereas scatter and attenuation corrected SPECT data will be referred to as SAC SPECT data.

2.3.3. Spatial normalisation: To perform SPECT measurements in the VOIs of the spatial model (see §2.1), the spatial normalisation method described by [Friston et al 1995] and implemented in the Statistical Parametric Mapping (SPM99 I) software was used. SPECT data and VOIs of the spatial model were both spatially normalised to a mean anatomical reference volume, represented by the T1 template provided by SPM, as follows:

- A non-linear geometric transformation was estimated to match the 3D T1-weighted MRI of the Zubal phantom, and thus the VOIs, on the T1 SPM template.

- For the 27 healthy subjects, SPECT images were spatially normalised to the SPECT template provided by SPM using an affine geometric transformation, as recommended by [Acton and Friston 1998] ; the SPECT SPM template is by construction in the same referential as the T1 SPM template.

- For the 10 MTLE patients, a two-step approach was used to achieve spatial normalisation of ictal SPECT data, which exhibited large hyperperfused areas. First, an inter-modality / intra-patient rigid registration was performed between each patient's ictal SPECT and MRI data, by maximisation of mutual information [Maes et al 1997]. Each subject's 3D T1-weighted MRI was then spatially normalised to the T1 SPM template, using a non-linear geometric transformation.

All these linear or non-linear geometric transformations allowed resampling of the SPECT data and the VOIs in the unique mean anatomical reference, i.e., the T1 SPM || SPM software: http://www.fil.ion.ucl.ac.uk/spm/ 
template, using trilinear interpolation. Spatial normalisation was visually checked by a neurosurgeon, by superimposing each SPECT, MRI and VOIs resampled in this mean anatomical reference volume (see Figure 2). No significant deformation errors were visually detected.

2.3.4. Intensity normalisation of the SPECT data: Because the injected radioactivity dose and resulting uptake varied from one patient to another, proportional scaling was used to remove the confounding effect of global inter-acquisition changes in SPECT [Acton and Friston 1998]: each SPECT data set was intensity normalised so that the mean voxel count was about 50 counts/voxel in a VOI including the whole brain (i.e. grey matter, white matter and ventricles). We chose the whole brain as the reference region for normalisation, as we could not apply the usual normalisation with respect to the mean cerebellum activity, due to its implication during temporal lobe seizures [Boussion et al 2000].

2.3.5. Measurement of perfusion on real SPECT data: For each spatially normalised VOI $i(i \in<1,40>)$ and each spatially normalised SPECT data set $j(j \in<1,27>$ for healthy subjects, $j \in<1,10>$ for MTLE patients), we estimated the mean $\bar{x}_{i j}$, the standard deviation $\hat{\sigma}_{i j}$, and the coefficient of variation $c v_{i j}=\hat{\sigma}_{i j} / \bar{x}_{i j}$ of the SPECT signal, within the considered VOI. The population mean $\bar{x}_{i}$, and associated standard deviation $\hat{\sigma}_{i}$ were then estimated from the distribution of the $\bar{x}_{i j}$ for each VOI $i$ over the entire set of SPECT data of a given group (subjects or patients).

To assess perfusion homogeneity among each VOI, the population mean coefficient of variation $\bar{c} \bar{v}_{i}$ was also estimated from the distribution of the $c v_{i j}$ for each VOI $i$. As proposed by [Lobaugh et al 2000], VOIs showing a mean $\overline{c v}_{i}$ greater than two standard deviations above the mean over all $c v_{i j}$ were considered non relevant to describe the studied perfusion pattern.

2.3.6. Partial volume effect correction: Because of the poor spatial resolution of SPECT, VOI measurements are biased by partial volume effect. For instance, the white matter VOI contains signal coming from the grey matter. To reduce the impact of partial volume effect upon perfusion measurements in white matter, four spherical VOIs with a radius of $7 \mathrm{~mm}$ were hand positioned in the centre of the white matter compartment of the Zubal phantom. Perfusion values obtained by averaging the SPECT signal from these four spheres are referred to as "WhiteMatVOI" values.

To correct regional mean values $\bar{x}_{i j}$ for partial volume effect on all VOIs for each subject, we applied a deconvolution method, named the Geometric Transfer Matrix (GTM) method, proposed for PET data by [Frouin et al 2002] and recently evaluated on ${ }^{123}$ I SPECT data [Soret et al 2003]. The GTM method assumes that the radiotracer activity distribution can be described as a set of $N$ VOIs, each with a homogeneous activity concentration $a_{i j}$. The mean value $\bar{x}_{i j}$ actually measured within the $i^{\text {th }}$ VOI is then assumed to be a weighted sum of the true underlying activities $a_{k j}$ of all 
anatomical structures within the neighborhood of this VOI. The $N \times N$ matrix $\mathbf{W}$ of these weights defining the Geometric Transfer Matrix was estimated as described in [Frouin et al 2002], given that all our reconstructed SPECT data had an estimated spatial resolution of $F W H M=12.2 \mathrm{~mm}$. Partial volume correction was then performed by inverting the GTM matrix W. $N=42$ anatomical structures were considered for partial volume correction including the skin, the fat, the skull and the cerebrospinal fluid as extra structures, in addition to those of the spatial model of brain anatomy. In the following, partial volume corrected SPECT measurements will be referred to as PVC SPECT measurements.

2.3.7. Statistical analysis of perfusion values: Distributions of $\bar{x}_{i j}$ measurements reflect the normal or the pathological perfusion patterns, characteristic of the selected sets of SPECT data. Both patterns were characterised by the average activity value $\bar{x}_{i}$ and associated standard deviation $\hat{\sigma}_{i}$ in each VOI. The effect of the anatomical structure, i.e. VOIs, over the perfusion measurements was assessed by analysis of variance. Such an analysis was performed to assess the relevance of the segmentation of the brain in VOIs, and more attention was paid to the analysis of the residuals than to the result of the $\mathrm{F}$ test.

For each pair of lateralised VOIs $i$, an inter-hemispheric asymmetry index was calculated as the relative difference between the left $\left(\bar{x}_{i, L}\right)$ and right $\left(\bar{x}_{i, R}\right)$ measurements with respect to the right hemisphere for healthy subject, i.e.: $\bar{x}_{i, \text { Diff }}=\left(\bar{x}_{i, L}-\right.$ $\left.\bar{x}_{i, R}\right) / \bar{x}_{i, R}$. Similarly, for MTLE patients, the asymmetry index was calculated as the relative difference between the pathological $\left(\bar{x}_{i, P}\right)$ and the healthy $\left(\bar{x}_{i, H}\right)$ hemisphere measurements with respect to the healthy hemisphere: $\bar{x}_{i, D i f f}=\left(\bar{x}_{i, P}-\bar{x}_{i, H}\right) / \bar{x}_{i, H}$. The significance of the asymmetry was tested using a non parametric paired t-test together with bootstrap resampling [Efron and Tibshirani 1993], to account for the small sample size and non Gaussian distribution of the VOI values. Considering a pvalue threshold of 0.05 for the t-test, we applied a Bonferroni correction for multiple comparison. VOIs were then considered asymmetric if the pvalue was less than $0.05 / 20=0.0025\left(N_{a}=20\right.$ being the number of tested VOIs for each subject's hemisphere).

For healthy subjects, analysis of SAC-PVC perfusion measurements was performed, whereas for MTLE patients we analysed AC-PVC perfusion measurements.

2.3.8. Derivation of activity maps: Following the statistical analysis of perfusion values, we assigned a relative mean perfusion value (in counts/voxel) to each VOI of the spatial model of brain anatomy, for a normal SPECT and an ictal SPECT. Normal activity map was set symmetrical, whereas for ictal SPECT, asymmetrical activity was set for VOIs with a significant inter-hemispheric asymmetry. 


\subsection{Realistic simulation of SPECT data}

The theoretical attenuation and ${ }^{99 m}$ Tc-HMPAO activity maps described above were used as an input of the Monte Carlo simulator SimSET 9 [Harrison et al 1993] to generate realistic SPECT data. Using SimSET, attenuation and Compton scatter were modelled through Monte Carlo methods, while the collimator and gamma camera responses were accounted for using analytical models. Because simulated SPECT projections are not Poisson distributed when an analytical model is used for collimation, we simulated a large number of photons to obtain almost noise-free projections. These projections were rescaled and Poisson noise was then added. Two SPECT simulations, corresponding respectively to the normal and the ictal perfusion models previously described (cf. $\S 2.3 .8)$, were thus generated.

For both SPECT simulations, $2.10^{9}$ photons were simulated. The number of "detected" photons after simulations was around 23 million, which corresponds to approximately five or six times more counts than in our real SPECT acquisitions (average number of counts was around 4.5 million). These simulations were then rescaled to obtain a total number of counts of 4.5 million. The intrinsic detector response was simulated by smoothing each projection using a 2D Gaussian filter at the intrinsic spatial resolution of the camera, i.e., $F W H M=3.5 \mathrm{~mm}$. Poisson noise was finally added. The geometric response of an ultra high-resolution parallel collimator identical to that used for the real acquisitions was simulated. Sixty-four projections over $360^{\circ}$ (matrix: $128 \times 128$, pixel size: $4.51 \mathrm{~mm}$ ) were simulated using a $20 \%$ energy window centred on $140 \mathrm{keV}(126-154 \mathrm{keV})$ and a $(111-125 \mathrm{keV})$ Compton window. Reconstruction was performed by filtered backprojection using a ramp filter (Nyquist frequency cutoff). As for the real data, the reconstructed data were post-filtered with an $8 \mathrm{~mm} \mathrm{FWHM} 3 \mathrm{D}$ Gaussian filter. By simulating the acquisition of a linear source in the air using similar acquisition and reconstruction characteristics, simulated SPECT spatial resolution was estimated to be $14.3 \mathrm{~mm}$ FWHM on reconstructed data.

\subsection{Evaluation of the relevance of simulated SPECT data}

Our goal was to check whether simulated SPECT agreed with the actual distribution of perfusion estimated from measurements on real SPECT data. Anatomical standardisation method was performed before VOI measurements on both normal and ictal simulated SPECT data. The same spatial and intensity normalisations as those used on real SPECT data were performed. Let $\bar{s}_{i}$ be the mean intensity measured on a simulated SPECT within the VOI $i$. Dif $f_{i}=\left(\bar{s}_{i}-\bar{x}_{i}\right) / \bar{x}_{i}$ denotes the relative difference of quantification of $\bar{s}_{i}$ when the mean perfusion value measured on real SPECT data $\bar{x}_{i}$ is considered as the reference measurement. To limit bias as much as possible, both simulated and real SPECT data were corrected for uniform attenuation, scatter and partial volume effect. SAC-PVC SPECT measurements were used to compare real and

I Simulation System for Emission Tomography (SimSET) package, Washington university: http://depts.washington.edu/ simset/html/simset_main.html 
simulated normal SPECT data, whereas AC-PVC SPECT measurements were used to compare real and simulated ictal SPECT data.

Since asymmetry measurements are a key issue to characterise normal and ictal perfusion patterns, we also measured inter-hemispheric asymmetry indexes obtained within simulated SPECT data: $\bar{s}_{i, D i f f}=\left(\bar{s}_{i, L}-\bar{s}_{i, R}\right) / \bar{s}_{i, R}$ for normal SPECT simulations and $\bar{s}_{i, D i f f}=\left(\bar{s}_{i, P}-\bar{s}_{i, H}\right) / \bar{s}_{i, H}$ for ictal SPECT simulations.

\section{Results}

\subsection{Perfusion model and simulation of normal SPECT}

The distributions of normal perfusion values estimated at each VOI from the 27 healthy subjects are represented using boxplot in Figure 3. For SPECT measurements performed on SAC-PVC post-corrected data, the empirical means $\bar{x}_{i}$ and the standard deviations $\hat{\sigma}_{i}$ of the perfusion values $\bar{x}_{i j}$ estimated within each VOI $i$ for the left hemisphere and the right hemisphere as well as inter-hemispheric asymmetry indexes are presented in Table 2. Analysis of variance showed a highly significant effect of the anatomical structure (i.e., VOIs) over the measured normal perfusion values $(F=116.1$, pvalue $<0.001)$. The relevance of the proposed VOIs segmentation to describe this perfusion pattern was confirmed as we measured a high adjusted determination coefficient $\left(R_{\text {adjust }}^{2}=\right.$ 0.81). Graphical analysis allowed us to check that the residuals were gaussian and homoscedastic, as classically assumed by the analysis of variance. As the GTM method corrected only mean perfusion values $\bar{x}_{i j}$ for partial volume effect, the coefficients of variation $c v_{i j}$ were estimated on SAC perfusion measurements without PVC. No mean coefficient of variation $\overline{c v}_{i}$ was greater than two standard deviations above the mean of all $c v_{i j}$, i.e., 0.29. The perfusion values within each VOI can thus be considered to be relatively homogeneous, confirming the consistency of the anatomic segmentation used for the data analysis. Note that SAC perfusion measurements within the WhiteMatVOI VOI, chosen to avoid partial volume effect within the white matter, were very similar to the SAC-PVC measurements within the White-Matter VOI.

A slight mean perfusion asymmetry, i.e. a mean $+2.0 \%$ hyperperfusion in the right hemisphere, was found significant in some cortical areas (see Table 2), namely: Cortex $(+2.0 \%$, non parametric paired t-test, pvalue $<0.0025)$, Frontal-Orbit $(+4.5 \%$, pvalue $<0.0025)$ and Parietal $(+3.5 \%$, pvalue $<0.0025)$.

SAC-PVC perfusion measurements within the 27 healthy subjects were used to generate an average theoretical model of normal perfusion, by assigning to each VOI the mean perfusion value $\bar{x}_{i}$ estimated over the population. The perfusion model is presented on Figure 4(a). By definition, the normal perfusion model was set completely symmetrical. Figure 4(b) presents the corresponding SPECT simulation after tomographic reconstruction and attenuation correction. 


\subsection{Perfusion model and simulation of ictal SPECT for mesial temporal lobe epilepsy}

The distributions of ictal perfusion values estimated at each VOI from the ten MTLE patients are represented using boxplot in Figure 5. For SPECT measurements performed on AC-PVC post-corrected data, the empirical means $\bar{x}_{i}$ and standard deviations $\hat{\sigma}_{i}$ of the perfusion values $\bar{x}_{i j}$ estimated within each VOI $i$ are shown in Table 3. Measurements for the diseased hemisphere and the healthy hemisphere as well as inter-hemispheric asymmetry indexes are presented. Analysis of variance showed a highly significant effect of the anatomical structure (i.e., VOIs) over the measured ictal perfusion values $\left(F=26.8\right.$, pvalue $<0.001$ and $R_{\text {adjust }}^{2}=0.72$ ). As for the normal perfusion study, the relevance of the proposed VOIs segmentation was confirmed for ictal data: high $R_{\text {adjust }}^{2}=0.72$ and graphical verification that residuals were gaussian and homoscedastic. Moreover, coefficients of variation $\overline{c v}_{i j}$, estimated from AC measurements without PVC, showed no mean $\bar{c} \bar{v}_{i}$ greater than two standard deviations above the mean of all $c v_{i j}$, i.e., 0.27. The distribution of the perfusion values was considered to be relatively homogeneous within each VOI, confirming again that the anatomic segmentation was appropriate for the analysis of ictal data.

Several perfusion asymmetries were proved highly significant in temporal areas, namely: Temporal-Lateral $(+18.1 \%$, non parametric paired t-test, pvalue $<0.0025)$, Temporal-Pole $(+27.2 \%$, pvalue $<0.0025)$ and Temporal-Mesial $(+6.7 \%$, pvalue $<$ 0.01). Significant hyperperfusion was also observed in the neighbourhood of the temporal lobe, namely: Insula $(+15.5 \%$, pvalue $<0.0025)$, Frontal-Orbit $(+14.8 \%$, pvalue $<0.0025)$, Lenticular-Nuclei $(+8.5 \%$, pvalue $<0.01)$, and a slightly significant hyperperfusion in the Cerebellum $(+5.0 \%$, pvalue $<0.05)$. All asymmetries consisted in hyperperfusion of the pathological hemisphere, slightly significant hypoperfusion of the pathological hemisphere was only observed in the Occipital-Mesial $(-9.8 \%$, pvalue $<0.01)$ and Frontal-Lateral $(-11.5 \%$, pvalue $<0.05)$ VOIs.

AC-PVC perfusion measurements within the 10 MTLE patients were used to generate an average theoretical model of ictal perfusion, by assigning to each VOI the mean perfusion value $\bar{x}_{i}$ estimated over the population. The VOIs with a significant asymmetry were considered to generate inter-hemispheric asymmetries within the average perfusion model. The ictal perfusion model is shown on Figure 6(a), and corresponding SPECT simulation is presented on Figure 6(b) after tomographic reconstruction and attenuation correction.

\subsection{Evaluation of the relevance of simulated SPECT data}

Visual comparison of both simulated and real SPECT data, spatially and intensity normalised, is shown to provide qualitative evaluation of the simulation realism, see Figures 4(b) and 4(c) for normal SPECT simulation and Figures 6(b) and 6(c) for ictal SPECT simulation. Only AC SPECT data are presented for this visual comparison.

SAC-PVC perfusion measurements obtained on the normal simulated SPECT as well as AC-PVC perfusion measurements obtained on ictal simulated SPECT are 
shown super-imposed on distributions of corresponding real perfusion measurements (see grey curves on Figures 3 and 5). The results show that the main trends of real perfusion distribution are respected by our simulated data. Main discrepancies were observed within the White-Matter, Temporal-Mesial, Frontal-Orbit, Frontal-Lateral and Occipital Lateral VOIs for both normal and ictal data, and within the Caudate-Nuclei VOI for ictal data only.

These results were confirmed by the estimation of relative quantification differences $\operatorname{Diff}_{i}$ for each VOI $i$, see Figure 7(a) for normal SAC-PVC SPECT measurements and Figure 7(b) for ictal AC-PVC SPECT measurements. In most of the VOIs, the differences $\operatorname{Diff}_{i}$ remained between $5 \%$ and $15 \%$. This means that regional measurements performed on the simulated images differed, on average, by less than $15 \%$ from corresponding measurements performed on real data. Differences greater than $20 \%$ were observed within the White-Matter and Occipital-Lateral VOIs for normal data and within the Caudate-Nuclei (from Healthy hemisphere) and Occipital-Lateral VOIs for ictal data.

Inter-hemispheric asymmetry indexes measured on normal and ictal simulated SPECT data are shown on Figures 8(a) and 8(b). Distribution of inter-hemispheric asymmetry indexes measured among real data (see column $\bar{x}_{i, \text { Diff }}$ from Tables 2 and 3) are also presented using boxplot representations. Even if the activity map of normal perfusion was set completely symmetrical before spatial normalisation, some asymmetries were measured on the normal simulated SPECT data, showing the largest asymmetries within the right Occipital-Lateral $(+12.4 \%)$, the right TemporalPole $(+9.5 \%)$ and the left Caudate-Nuclei $(+7.4 \%)$ VOIs. Among all other VOIs, asymmetries lower than $5 \%$ were observed. These asymmetries probably result from segmentation errors introduced by the spatial normalisation procedure and their impact on PVC. We thus have to consider carefully the significant asymmetries found when analysing SPECT data from the 27 healthy subjects. On the other hand, main relative asymmetry measurements performed on the ictal simulated SPECT were slightly underestimated compared to mean measurements on real ictal SPECT data but remained within the range of the distributions (see Figure 8(b)). Overall, simulated asymmetries mimick the main trends of real perfusion values distributions quite realistically, and especially reproduce well the hyperperfusion of the Temporal-Pole VOI and the hypoperfusion of the Frontal-Lateral and Occipital-Mesial VOIs.

\section{Discussion}

We have presented a generic methodology for generating validation data sets dedicated to the evaluation of SPECT/MRI fusion methods. The originality of this approach is that a method of inter-individual analysis of SPECT data by anatomic standardisation was coupled with a realistic modelling of the physics of SPECT using Monte Carlo techniques. The SPECT simulations presented here have already been used to evaluate and compare MRI/SPECT registration methods [Grova et al 2002]. 


\subsection{Anatomic standardisation analysis}

To model perfusion patterns from real SPECT data, we proposed a VOI-based anatomical standardisation approach. The importance of an appropriate segmentation of the brain for inter-individual analysis of brain function has been underlined previously [Lobaugh et al 2000, Van Laere et al 2001, Kang et al 2001]. Our definition of the spatial model of brain anatomy results from a compromise between SPECT spatial resolution and "a priori" knowledge of the anatomy and physiology of the studied phenomena. We segmented different parts of the cerebral lobes (especially the temporal and frontal lobes), known to exhibit characteristic patterns of ictal perfusion in temporal lobe epilepsy [Ho et al 1996]. Our segmentation of the temporal lobe was based on electrophysiological criteria [Bartolomei et al 1999] and differed from other spatial models deduced from sulco-gyral morphology [Lobaugh et al 2000, Kang et al 2001]. Analysis of the coefficients of variation observed for each VOI as well as of the residuals of the variance analysis, both suggested the relevance of the proposed segmentation.

Compensation of inter-individual anatomical variability was achieved using the spatial normalisation method proposed by [Friston et al 1995]. This method was proved to be very accurate, especially to preserve sulco-gyral morphology [Hellier et al 2002]. Because of the impact of SPECT hypoperfusion areas on non-linear spatial normalisation accuracy [Acton and Friston 1998, Stamatakis et al 2001], affine geometrical transformations were chosen to normalise the SPECT from the 27 healthy subjects and a two-step approach dedicated to ictal SPECT normalisation was proposed. Although the value of this two-step approach should be further characterised, it was considered as sufficiently accurate from visual verification by a neurosurgeon. Because of the spatial normalisation step and the low spatial resolution of the SPECT images, we assumed in the present study that the anatomical inter-individual variability had a negligible impact on the definition of the SPECT perfusion model.

\subsection{Realistic SPECT simulations for evaluation purposes}

To simulate realistic brain perfusion SPECT data, not only the initial activity distribution has to be consistent with the actual distribution of the tracer, but the model used to create the SPECT images should also be precise enough. We therefore used Monte Carlo simulations that enable a precise modelling of all physical and geometrical processes involved in SPECT.

An alternative would be to use real acquisitions of physical phantoms, e.g., [Hoffman et al 1990]. Although such phantoms can provide an absolute gold standard to evaluate registration methods, they cannot model complex pathological perfusion patterns, due to the limited number of compartments that they include (grey matter, white matter, and cerebrospinal fluid). Complex perfusion patterns can be modelled by artificially modifying local activity of real SPECT images, e.g., [Brinkmann et al 1999, Stamatakis et al 2001]. Such simulations provide an absolute gold standard for localisation of simulated perfusion abnormalities, but they no longer take into account 
the physics of data generation and acquisition at the level of these defects. Bias may be induced by artificial contours of such simulated abnormalities. Analytical (as opposed to Monte Carlo) SPECT simulations have also been used to simulate brain perfusion scans (see for instance [Lahorte et al 2000, Thurjfell et al 2000]). However, because some phenomena cannot be accurately modelled analytically, Monte Carlo simulations are widely recognized to be a more powerful tool to accurately model SPECT data [Zaidi 1999]. Our methodology is similar to approaches where realistic simulations were obtained by defining an activity map at the resolution of an accurately segmented and labelled MRI [Zubal et al 1994, Collins et al 1998, El Fakhri et al 2001]. [Collins et al 1998] proposed such a spatial model of brain anatomy to create a simulator of MRI data ${ }^{+}$and PET data but did not propose any method to initialise the activity map. Using SimSET and the Zubal phantom to simulate normal SPECT, [El Fakhri et al 2001] initialised their activity map using average normal perfusion values measured by [Koyama et al 1997]. Our methodology describes how to generate a perfusion model and corresponding simulations specific to a clinical context, especially when no perfusion model is available in the literature.

\subsection{Clinical relevance of normal and pathological perfusion models and simulations}

Our methodology yielded two distributions of relative values of perfusion, characteristic of normal perfusion and ictal perfusion as seen in MTLE. To derive the activity values in the different compartments of our perfusion model, uniform attenuation correction has been considered sufficient [Iida et al 1998]. Scatter correction was performed on SPECT images from healthy subjects only. However, only slight differences were observed when comparing relative differences Dif $f_{i}$ measured on normal simulated SPECT obtained using perfusion models deduced from $\mathrm{AC}$ and $\mathrm{SAC}$ perfusion measurements (results not shown). Scatter correction was therefore not considered to be crucial to derive realistic perfusion models. Partial volume effect is expected to generate relative quantification error up to $50 \%$ for internal structures [Soret et al 2003]. To derive a perfusion model as accurate as possible, perfusion measurements were then corrected for partial volume effect using the GTM approach proposed for PET data by [Frouin et al 2002] and evaluated on ${ }^{123}$ I SPECT data by [Soret et al 2003]. Relative quantification differences Dif $f_{i}$ found between real and simulated data remained lower than $20 \%$ for almost all anatomical structures (cf. Figures 7(a) and 7(b)). These differences Diff $f_{i}$ as well as the inter-hemispheric asymmetry indexes estimated on simulated data (see §3.3) suggest that our perfusion model and corresponding simulated SPECT are realistic enough for the assessment of SPECT/MRI fusion methods. Moreover, acquiring more normal and ictal SPECT data on the same machine would also improve the accuracy of our perfusion models.

Validation of the accuracy of the different correction methods considered to derive our perfusion models was beyond the scope of this study. In particular, the accuracy + MRI simulator: http://www.bic.mni.mcgill.ca/brainweb/ 
of the GTM approach for partial volume correction has only been evaluated so far by taking into account few anatomical structures (7 VOIs for [Frouin et al 2002] and 5 VOIs for [Soret et al 2003]) and by studying the impact of linear registration errors. In our approach, SPECT data need to be spatially normalised (using non linear registration) before VOIs segmentation and 42 VOIs were used for deconvolution. We believe that the largest quantitative differences Diff $f_{i}$ (see Figures $7(\mathrm{a})$ and $7(\mathrm{~b})$ ) and inter-hemispheric asymmetries (see Figure 8(a)) found for the Occipital-Lateral or Caudate-Nuclei VOIs are due to spatial normalisation errors and thus segmentation errors within these structures. Indeed, segmentation errors were shown to greatly affect the accuracy of the GTM method, especially when the volume of the structure is under-estimated [Frouin et al 2002, Soret et al 2003]. Further investigations should then study the reliability of the GTM correction in perfusion SPECT when using anatomic standardisation approaches for VOIs segmentation and a large number of anatomical structures for deconvolution.

To model brain perfusion in SPECT, inter-individual functional variability of normal or pathological perfusion should be taken into account. In our work, we modelled only the average perfusion measured in a set of normal or ictal SPECT images and used these average models to perform SPECT simulations. The complexity of characterising a normal perfusion pattern and the effects of the healthy subject selection and acquisition protocols on perfusion measurements have already been highlighted [Van Laere et al 2001, Pagani et al 2002]. Using linear models [Van Laere et al 2001, Pagani et al 2002] and principal component analysis [Pagani et al 2002], these authors investigated different properties of normal perfusion measurements, such as variability, inter-hemispheric asymmetry, effect of age and gender. These results may be easily included in our perfusion models. A more complete analysis of the population perfusion values distribution and of its spatial correlation was beyond the scope of this work but is under investigation. The characterisation of inter-individual functional variability from VOI-based measurements may be later used to generate large validation data sets accounting for physiological and physio-pathological patients' variability.

Finally, our normal perfusion model could be compared with other models of normal SPECT perfusion [Koyama et al 1997, Lobaugh et al 2000, Van Laere et al 2001, Pagani et al 2002]. MTLE is associated with a particular pattern of ictal SPECT perfusion [Ho et al 1996]. To our knowledge, there are no studies regarding characteristic ictal perfusion measurements for MTLE based on a non-supervised analysis from an adequate anatomic segmentation for temporal lobe epilepsy.

\section{Conclusion}

The methodology for realistic SPECT simulation presented here enables generation of fully controlled data for assessing processing methods involving SPECT/MRI image fusion, within the normal or pathological clinical setting. The degree of realism necessary to create a theoretical model of perfusion and to perform relevant simulations can 
be adjusted as a function of the evaluation objectives. Our approach has already been used to study the behaviour and performance of registration methods based on statistical similarity in the context of ictal SPECT [Grova et al 2002]. Other potential applications are the assessment of methods of ictal/interictal SPECT subtraction, or of non-supervised inter-hemispheric analysis (e.g. [Aubert-Broche et al 2003]). In the context of epilepsy, we proposed a theoretical model of ictal perfusion resulting from an inter-individual analysis guided by the anatomic segmentation recognized by epilepsy specialists. The model of brain anatomy presented here is generic and could be adapted to other studies concerning temporal or frontal lobe epilepsy, or other diffuse diseases of the brain.

\section{Acknowledgements}

This work was partly supported by grants from the "Ligue Française Contre l'Epilepsie" and from the "Conseil Régional de Bretagne". We also would like to thank Dr. Leighton Barnden Ph.D. (Woodville Hospital, Nuclear Medicine Department, Australia) for providing normal SPECT data sets, Anthonin Reilhac (Montreal Neurological Institute, Canada) for providing the GTM method for partial volume correction and Sébastien Hapdey (INSERM U494, Paris and NIH Bethesda MD, USA) for fruitful discussion concerning SPECT simulations on SimSET.

\section{References}

[Acton and Friston 1998] Acton P and Friston K 1998 Statistical Parametric Mapping in Functional Neuroimaging: Beyond PET and fMRI Activation Studies Eur. J. Nucl. Med. 25 663-667

[Aubert-Broche et al 2003] Aubert-Broche B, Grova C, Jannin P, Buvat I, Benali H and Gibaud B 2003 Detection of Inter-Hemispheric Asymmetries of Brain Perfusion in SPECT Phys. Med. Biol. 48 1505-1517

[Barnden et al 2002] Barnden L, Behin-Ain S and Unger S 2002 Optimisation of Brain SPECT Accuracy by Comparison of Hoffman Phantom SPECT Images with the Numerical Hoffman Template ANZ Nuclear Medicine p 8, abstract

[Bartolomei et al 1999] Bartolomei F, Wendling F, Vignal J P, Kochen S, Bellanger J J, Badier J M, Le Bouquin-Jeannes R and Chauvel P 1999 Seizures of Temporal Lobe Epilepsy: Identification of Subtypes by Coherence Analysis Using Stereo-Electro-Encephalography Clin. Neurophysiol. $1101741-1754$

[Boussion et al 2000] Boussion N, Ryvlin P, Isnard J, Houzard C, Maugière F and Cinotti L 2000 Towards an Optimal Reference Region in Single-Photon Emission Tomography Difference Images in Epilepsy Eur. J. Nucl. Med. 27 155-160

[Brinkmann et al 1999] Brinkmann B H, O’Brien T J, Aharon S, O'Connor M K, Mullan B P, Hanson D P and Robb R A 1999 Quantitative and Clinical Analysis of SPECT Image Registration for Epilepsy Studies J Nucl. Med. 40 1098-1105

[Chang 1978] Chang L T 1978 A Method for Attenuation Correction in Radionucleide Computed Tomography IEEE Trans. Nucl. Sci. 25 638-643

[Collins et al 1998] Collins D L, Zijdenbos A P, Kollokian V, Sled J, Kabani N J, Holmes C J and Evans A C 1998 Design and Construction of a Realistic Digital Brain Phantom IEEE Trans. Med. Imaging 17 463-468 
[Devous et al 1998] Devous M, Thisted R, Morgan G, Leroy R and Rowe C 1998 SPECT Brain Imaging in Epilepsy: A Meta-Analysis J. Nucl. Med. 39 285-293

[Efron and Tibshirani 1993] Efron B and Tibshirani R 1993 An Introduction to the Bootstrap number 57 in Monographs on Statistics and Applied Probability (Boca Raton: Chapman and Hall/CRC)

[El Fakhri et al 2001] El Fakhri G, Moore S, Maksud P, Aurengo A and Kijewski M 2001 Absolute Activity Quantitation in Simultaneous 123I/99mTc Brain SPECT J. Nucl. Med. 42 300-308

[Friston et al 1995] Friston K, Ashburner J, Poline J, Frith C, Heather J and Frackowiak R 1995 Spatial Registration and Normalization of Images Hum. Brain Mapp. 2 165-189

[Frouin et al 2002] Frouin V, Comtat C, Reilhac A and Grégoire M C 2002 Correction of PartialVolume Effect for PET Striatal Imaging: Fast Implementation and Study of Robustness $J$. Nucl. Med. 43 1715-1726

[Grova et al 2002] Grova C, Jannin P, Biraben A, Buvat I, Benali H, Bernard A, Scarabin J and Gibaud B 2002 Validation of MRI/SPECT similarity-based registration methods using realistic simulations of normal and pathological SPECT data Computer Assisted Radiology and Surgery 2002, Paris (Berlin: Springer) pp 450-455

[Harrison et al 1993] Harrison R, Vannoy S, Haynor D, Gillispie S, Kaplan M and Lewellen T 1993 Preliminary Experience with the Photon History Generator Module of a Public-Domain Simulation System for Emission Tomography Conf. Rec. Nucl. Sci. Symp. volume 2 pp 11541158

[Hellier et al 2002] Hellier P, Ashburner J, Corouge I, Barillot C and Friston K 2002 Inter-Subject Registration of Functional and Anatomical Data Using SPM Lecture Notes in Computer Science (MICCAI 2002, Tokyo) (Berlin: Springer) pp 590-597

[Ho et al 1996] Ho S, Berkovic S, McKay W, Kalnins R and Bladin P 1996 Temporal Lobe Epilepsy Subtypes: Differential Patterns of Cerebral Perfusion on Ictal SPECT Epilepsia 37 788-795

[Hoffman et al 1990] Hoffman E, Cutler P, Digby W and Mazziotta J 1990 3-D Phantom to Simulate Cerebral Blood Flow and Metabolic Images for PET IEEE Trans. Nucl. Sci. 37 616-620

[Iida et al 1998] Iida H, Narita Y, Kado H, Kashikura A, Sugawara S, Shoji Y, Kinoshita T, Ogawa T and Eberl S 1998 Effect of Scatter and Attenuation Correction on Quantitative Assessment of Regional Cerebral Blood Flow with SPECT J. Nucl. Med. 39 181-189

[Jaszczak et al 1985] Jaszczak R, Floyd C and Coleman R 1985 Scatter Compensations Techniques for SPECT IEEE Trans. Nucl. Sci. 32 786-793

[Kang et al 2001] Kang K, Lee D, Cho J, Lee J, Yeo J, Lee S.K. Chung J K and Lee M 2001 Quantification of F-18 FDG PET Images in Temporal Lobe Epilepsy Patients Using Probabilistic Brain Atlas Neuroimage 14 1-6

[Koyama et al 1997] Koyama M, Kawashima R, Ito H, Ono S, Sato K, Goto R, Kinomura S, Yoshioka S, Sato T and Fukuda H 1997 SPECT Imaging of Normal Subjects with Technetium-99m-HMPAO and Technetium-99m-ECD J. Nucl. Med. 38 587-592

[Lahorte et al 2000] Lahorte P, Vandenberghe S, Van Laere K, Audenauert K, Lemahieu I and Dierckx R 2000 Assessing the Performance of SPM Analyses of SPECT Neuroactivation Studies Neuroimage 12 757-764

[Lee et al 2000] Lee J, Kim H J, Lee B, Kim O, Jeon T and Kim M 2000 Evaluation of Ictal Brain SPET Using Statistical Parametric Mapping in Temporal Lobe Epilepsy Eur. J. Nucl. Med. 27 $1658-1665$

[Lobaugh et al 2000] Lobaugh N, Caldwell C, Black S, Leibovitch F and Swartz S 2000 Three Brain SPECT Region-of-Interest Templates in Elderly People: Normative Values, Hemispheric Asymmetries, and a Comparison of Single- and Multihead Cameras J. Nucl. Med. 41 45-56

[Maes et al 1997] Maes F, Collignon A, Vandermeulen D, Marchal G and Suetens P 1997 Multimodality Image Registration by Maximization of Mutual Information IEEE Trans. Med. Imaging 16 187198

[O’Brien et al 1998] O'Brien T J, O'Connor M K, Mullan B P, Brinkmann B H, Hanson D, Jack C R 
and So E L 1998 Subtraction Ictal SPET Co-Registered to MRI in Partial Epilepsy: Description and Technical Validation of the Method with Phantom and Patient Studies Nucl. Med. Commun. $1931-45$

[Pagani et al 2002] Pagani M, Salmaso D, Jonsson C, Hatherly R, Jacobsson H, Larsson S and Wägner A 2002 Regional Cerebral Blood Flow Assessment by Principal Component Analysis and 99mTcHMPAO SPET in Healthy Subjects at Rest: Normal Distribution and Effect of Age and Gender Eur. J. Nucl. Med. 29 67-75

[Patterson and Wyper 1997] Patterson J and Wyper D 1997 SPECT Imaging of the Brain: Basics of SPECT (Dordrecht: Kluwer) chapter $1 \mathrm{R}$. Duncan edition pp 1-42

[Soret et al 2003] Soret M, Koulibaly P, Darcourt J, S. H and Buvat I 2003 Quantitative Accuracy of Dopaminergic Neurotransmission Imaging with 123-I SPECT J. Nucl. Med. 44 1184-1193

[Stamatakis et al 2001] Stamatakis E, Wilson J and Wyper D 2001 Spatial Normalization of Lesioned HMPAO-SPECT Images Neuroimage 14 844-852

[Talairach and Tournoux 1988] Talairach J and Tournoux P 1988 Co-Planar Stereotactic Atlas of the Human Brain (Stuttgard: Georg Thieme Verlag)

[Thurjfell et al 2000] Thurjfell L, Lau Y, Andersson J and Hutton B 2000 Improved Efficiency for MRI-SPET Registration Based on Mutual Information Eur. J. Nucl. Med. 27 847-856

[Van Laere et al 2001] Van Laere K, Versijpt J, Audenauert K, Koole M, Goethals I, Achten E and Dierckx R 2001 99mTc-ECD Brain Perfusion SPET: Variability, Asymmetry and Effect of Age and Gender in Healthy Adults Eur. J. Nucl. Med. 28 873-887

[Zaidi 1999] Zaidi H 1999 Relevance of Accurate Monte Carlo Modeling in Nuclear Medical Imaging Med. Phys. 26 574-608

[Zubal et al 1994] Zubal I, Harrell C, Smith E, Rattner Z, Gindi G and Hoffer P 1994 Computerized Three-Dimensional Segmented Human Anatomy Med. Phys. 21 299-302

[Zubal et al 1995] Zubal I, Spencer S, Imam K, Seibyl J, Smith E, Wisniewski G and Hoffer P 1995 Difference Images Calculated from Ictal and Interictal Technetium-99m-HMPAO SPECT Scans of Epilepsy J. Nucl. Med. 36 684-689 
Table 1. Correspondance between the VOIs of the Zubal phantom (columns) and some VOIs of our spatial model of brain anatomy (rows) - 'o': fusion of the Zubal phantom VOIs ' $x$ ': segmentation of the Zubal phantom VOI using the Talairach stereotactic atlas (Amyg.: Amygdala, Hipp.: Hippocampus, Temp.: Temporal Lobe, Front.: Frontal Lobe, Par.: Parietal Lobe, Occ.: Occipital Lobe, Put.: Putamen, Pall.: Pallidum) ; For instance, we defined the 'Cortex' VOI as the fusion of the VOIs 'Temp', 'Front', 'Par' and 'Occ', whereas the 'Temp' VOI of Zubal phantom was segmented to provide the VOIs 'Temporal-Pole', 'Temporal-Mesial', 'TemporalLateral' and 'Temporal-Posterior'

\begin{tabular}{|c|c|c|c|c|c|c|c|c|c|}
\hline Brain Entity & Amyg. & Hipp. & Temp. & Front. & Par. & Occ. & Put. & Pall. \\
\hline Cortex & & & 0 & 0 & 0 & 0 & & \\
\hline Healthy-Cortex & & & & 0 & 0 & 0 & & \\
\hline Lenticular-Nuclei & & & & & & & 0 & 0 \\
\hline Temporal-Pole & & & $\times$ & & & & & \\
\hline Temporal-Mesial & 0 & 0 & $\times$ & & & & & \\
\hline Temporal-Lateral & & & $\times$ & & & & & \\
\hline Temporal-Posterior & & & $\times$ & & & & & \\
\hline Frontal-Pole & & & & $\times$ & & & & \\
\hline Frontal-Orbit & & & & $\times$ & & & & \\
\hline Frontal-Mesial & & & & $\times$ & & & & \\
\hline Frontal-Lateral & & & & $\times$ & & & & \\
\hline Occipital-Mesial & & & & & & $\times$ & & \\
\hline Occipital-Lateral & & & & & & $\times$ & & \\
\hline
\end{tabular}

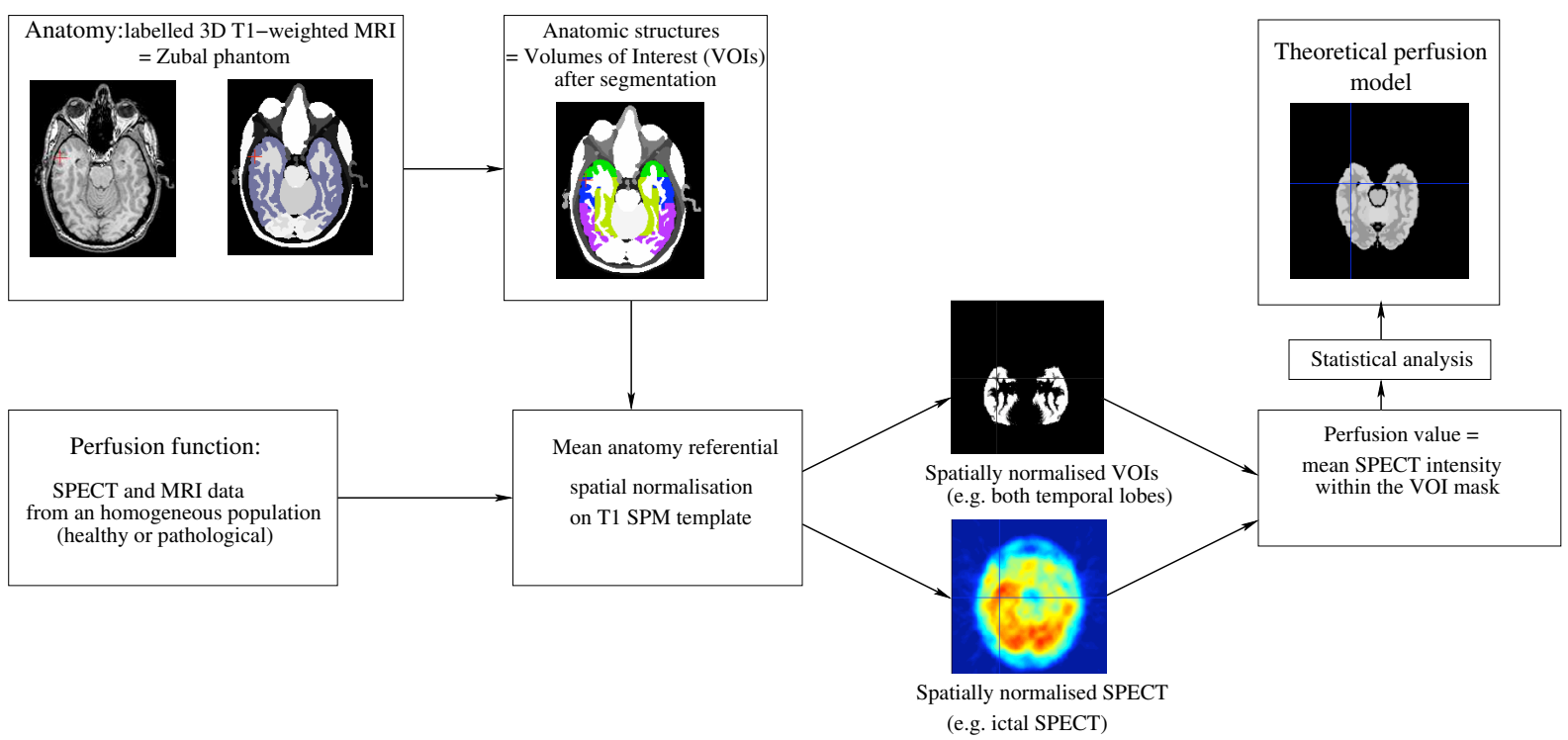

Figure 1. Construction of a theoretical perfusion model from real SPECT data 

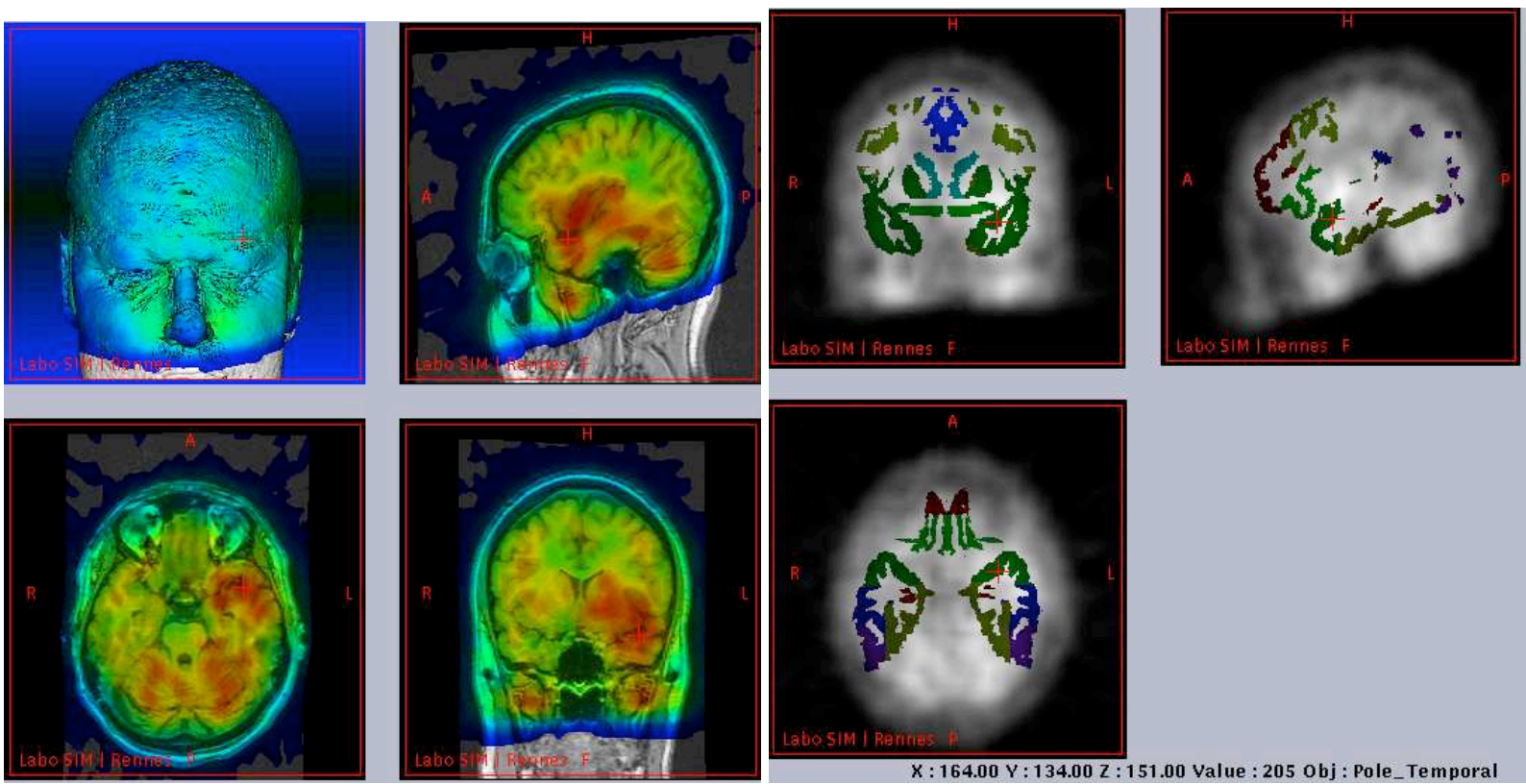

(a)

(b)

Figure 2. Multimodal data fusion for anatomical standardisation analysis: (a) ictal SPECT of a MTLE patient superimposed on its MRI, both being spatially normalised to the T1 SPM template, (b) Frontal, temporal and some internal VOIs of the spatial model of brain anatomy superimposed on the spatially normalised ictal SPECT of the same patient 
Table 2. Perfusion values obtained in 27 healthy subjects from SAC-PVC SPECT measurements (SAC measurements only for the WhiteMatVOI VOI) ; Empirical means $\bar{x}_{i}$ and standard deviations $\hat{\sigma}_{i}$ of the perfusion values $\bar{x}_{i j}$ estimated within each VOI $i$ in the left hemisphere $\left(\bar{x}_{i, L}\right)$ and the right hemisphere $\left(\bar{x}_{i, R}\right)$; Inter-hemispheric asymmetry indexes measured on real SPECT data $\left(\bar{x}_{i, D i f f}=\left(\bar{x}_{i, L}-\bar{x}_{i, R}\right) / \bar{x}_{i, R}\right)$; VOIs considered as significantly asymmetric are designed by: ${ }^{*} *$ ' $=($ pvalue $<0.05)$, $\left.{ }^{(* *}\right)=($ pvalue $<0.01),{ }^{(* * *)}=($ pvalue $<0.0025)$

\begin{tabular}{|c|c|c|c|}
\hline Brain Entity & $\bar{x}_{i, L}\left(\hat{\sigma}_{i, L}\right)$ & $\bar{x}_{i, R}\left(\hat{\sigma}_{i, R}\right)$ & $\begin{array}{l}\bar{x}_{i, D i f f}\left(\hat{\sigma}_{i, D i f f}\right) \\
\text { in } \%\end{array}$ \\
\hline Cortex & $57.3(1.10)$ & $58.5(1.34)$ & $-1.99(1.78)^{* * *}$ \\
\hline Healthy-Cortex & $59.7(1.65)$ & $60.6(2.00)$ & $-1.54(2.77)^{*}$ \\
\hline White-Matter & $32.0(2.05)$ & $31.5(2.06)$ & $1.53(3.06)$ \\
\hline WhiteMatVOI & $37.5(2.922)$ & $36.2(3.320)$ & $4.07(9.58)$ \\
\hline Caudate-Nuclei & $54.9(6.01)$ & $56.1(8.34)$ & $-0.68(13.98)$ \\
\hline Lenticular-Nuclei & $63.2(5.09)$ & $63.5(4.18)$ & $0.00(10.02)$ \\
\hline Thalamus & $62.4(6.43)$ & $65.4(5.25)$ & $-4.30(9.39)^{*}$ \\
\hline Insula & $66.8(5.98)$ & $66.4(6.05)$ & $1.23(11.93)$ \\
\hline Temporal-Pole & $48.7(5.07)$ & $50.7(4.25)$ & $-3.91(8.06)$ \\
\hline Temporal-Mesial & $53.4(3.02)$ & $54.7(2.25)$ & $-2.22(5.62)^{*}$ \\
\hline Temporal-Lateral & $56.5(3.11)$ & $58.3(2.94)$ & $-3.07(5.25)^{*}$ \\
\hline Temporal-Posterior & $54.0(3.05)$ & $55.0(2.57)$ & $-1.79(4.69)$ \\
\hline Frontal-Pole & $58.5(2.44)$ & $58.6(2.90)$ & $-0.07(3.62)$ \\
\hline Frontal-Orbit & $66.9(3.37)$ & $70.2(3.87)$ & $-4.47(4.60)^{* * *}$ \\
\hline Frontal-Mesial & $54.8(5.01)$ & $56.0(3.30)$ & $-2.14(7.56)$ \\
\hline Frontal-Lateral & $59.9(3.35)$ & $61.8(3.32)$ & $-3.04(4.24)^{*}$ \\
\hline Occipital-Mesial & $72.5(4.35)$ & $72.6(3.41)$ & $-0.18(4.79)$ \\
\hline Occipital-Lateral & $51.7(6.14)$ & $49.8(7.33)$ & $5.92(20.16)$ \\
\hline Parietal & $53.4(1.78)$ & $55.4(1.95)$ & $-3.52(2.71)^{* * *}$ \\
\hline Cerebellum & $56.6(2.95)$ & $57.1(2.67)$ & $-0.80(3.09)$ \\
\hline
\end{tabular}




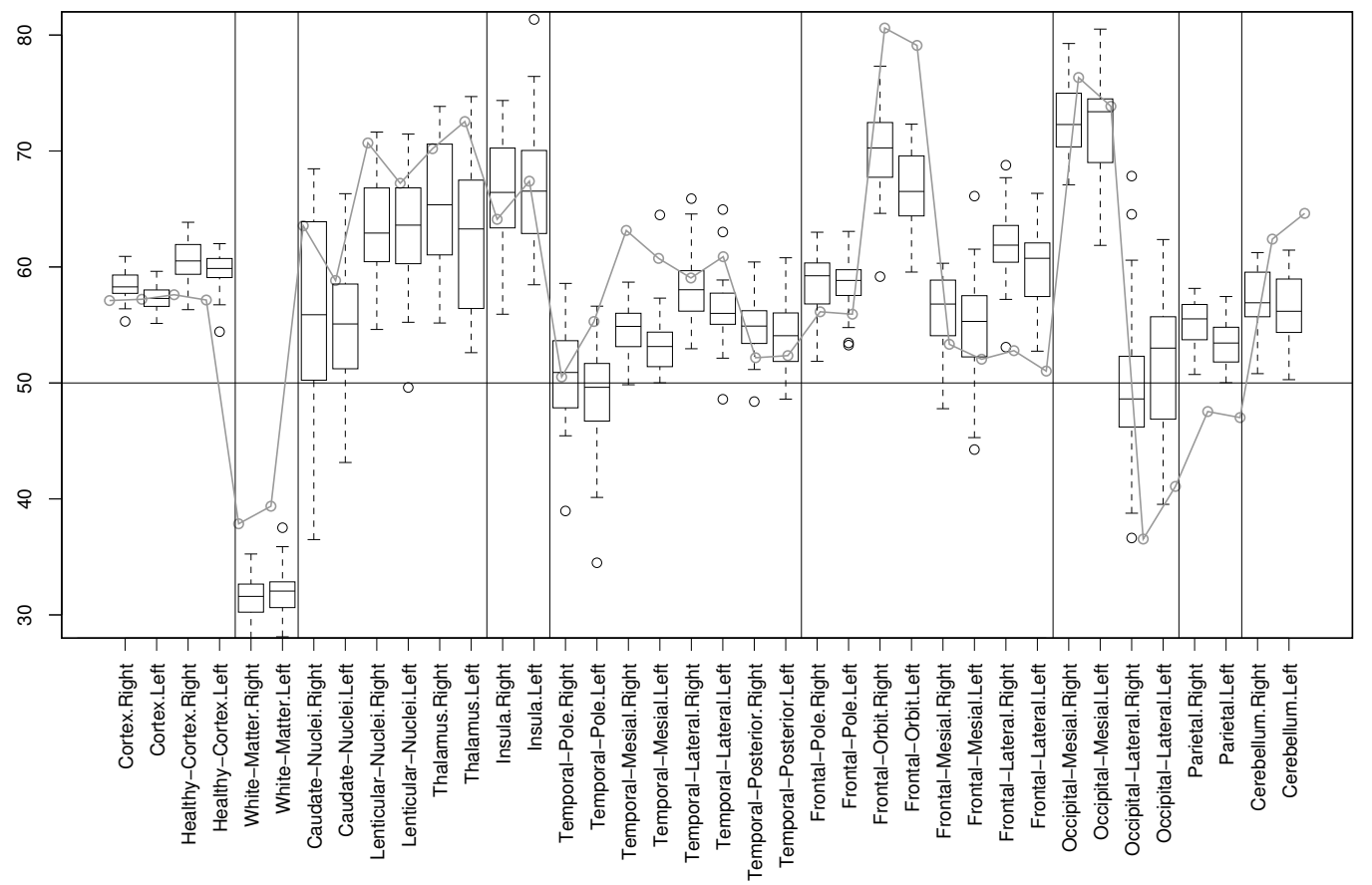

Figure 3. Distribution of SAC-PVC perfusion measurements at each VOI from the 27 healthy subjects using boxplot representations ; The grey curve indicates SACPVC perfusion measurements performed on the normal simulated SPECT (horizontal straight line $=$ activity of 50 counts/voxel)
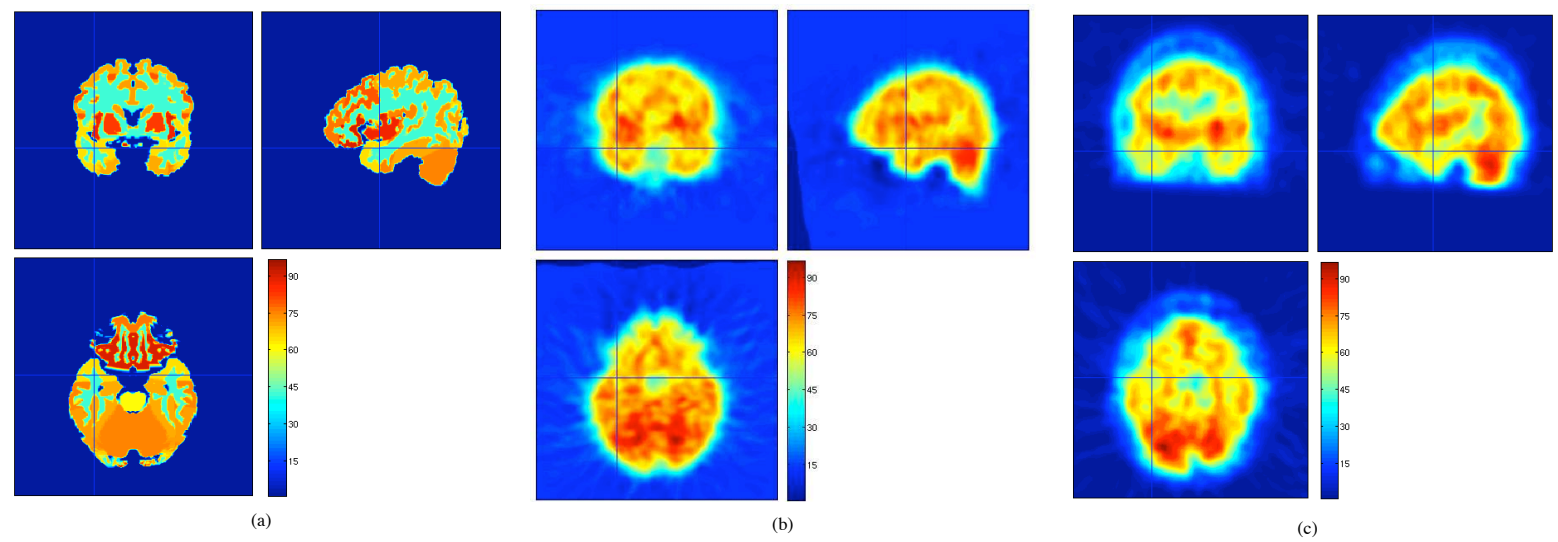

Figure 4. (a) Average theoretical model of normal perfusion; (b) Images reconstructed from the simulation of a normal SPECT perfusion scan ; (c) Example of real SPECT data from one healthy subject (both real and simulated SPECT being only corrected for attenuation) 
Table 3. Ictal perfusion values obtained in 10 MTLE patients from AC-PVC SPECT measurements (AC measurements only for the WhiteMatVOI VOI) ; Empirical means $\bar{x}_{i}$ and standard deviations $\hat{\sigma}_{i}$ of the perfusion values $\bar{x}_{i j}$ estimated within each VOI $i$ in the pathological hemisphere $\left(\bar{x}_{i, P}\right)$ and the healthy hemisphere $\left(\bar{x}_{i, H}\right)$ ; Inter-hemispheric asymmetry indexes measured on real SPECT data $\left(\bar{x}_{i, D i f f}=\right.$ $\left.\left(\bar{x}_{i, P}-\bar{x}_{i, H}\right) / \bar{x}_{i, H}\right)$; VOIs considered as significantly asymmetric are designed by: $\left.{ }^{*} *\right)=($ pvalue $<0.05),{ }^{(* *)}=($ pvalue $<0.01),{ }^{(* * *)}=($ pvalue $<0.0025)$

\begin{tabular}{|c|c|c|l|}
\hline Brain Entity & $\bar{x}_{i, P}\left(\hat{\sigma}_{i, P}\right)$ & $\bar{x}_{i, H}\left(\hat{\sigma}_{i, H}\right)$ & $\begin{array}{l}\bar{x}_{i, \text { Diff }}\left(\hat{\sigma}_{i, \text { Diff }}\right) \\
\text { in \% }\end{array}$ \\
\hline Cortex & $55.7(2.40)$ & $52.4(1.51)$ & $5.74(5.23)^{* * *}$ \\
\hline Healthy-Cortex & $51.0(3.26)$ & $51.0(2.34)$ & $-0.30(6.27)$ \\
\hline White-Matter & $41.3(3.59)$ & $41.8(3.28)$ & $-1.48(4.77)$ \\
\hline WhiteMatVOI & $44.2(3.40)$ & $44.3(3.53)$ & $0.045(6.80)$ \\
\hline Caudate-Nuclei & $56.0(11.72)$ & $56.5(7.82)$ & $-2.56(13.11)$ \\
\hline Lenticular-Nuclei & $67.3(2.50)$ & $61.6(5.06)$ & $8.48(7.22)^{* *}$ \\
\hline Thalamus & $65.9(8.23)$ & $61.5(13.10)$ & $6.962(13.78)$ \\
\hline Insula & $70.5(6.38)$ & $59.1(4.63)$ & $15.47(11.83)^{* * *}$ \\
\hline Temporal-Pole & $74.9(6.25)$ & $54.5(6.46)$ & $27.20(6.73)^{* * *}$ \\
\hline Temporal-Mesial & $63.3(2.87)$ & $59.0(3.39)$ & $6.73(4.11)^{* *}$ \\
\hline Temporal-Lateral & $66.0(7.62)$ & $53.4(2.19)$ & $18.11(9.75)^{* * *}$ \\
\hline Temporal-Posterior & $51.3(6.56)$ & $52.2(2.57)$ & $-3.49(15.99)$ \\
\hline Frontal-Pole & $46.5(3.86)$ & $49.0(3.77)$ & $-5.62(8.10)$ \\
\hline Frontal-Orbit & $71.0(5.47)$ & $60.3(4.25)$ & $14.80(7.23)^{* * *}$ \\
\hline Frontal-Mesial & $48.5(4.66)$ & $46.8(4.40)$ & $3.30(7.34)$ \\
\hline Frontal-Lateral & $47.4(6.58)$ & $52.0(3.99)$ & $-11.48(16.11)^{*}$ \\
\hline Occipital-Mesial & $58.7(5.26)$ & $64.0(4.38)$ & $-9.79(11.72)^{* *}$ \\
\hline Occipital-Lateral & $39.8(9.61)$ & $37.6(5.63)$ & $-1.04(35.28)$ \\
\hline Parietal & $44.8(3.73)$ & $47.3(3.29)$ & $-5.78(6.85)$ \\
\hline Cerebellum & $63.2(3.37)$ & $60.0(3.00)$ & $4.98(4.23)^{*}$ \\
\hline
\end{tabular}




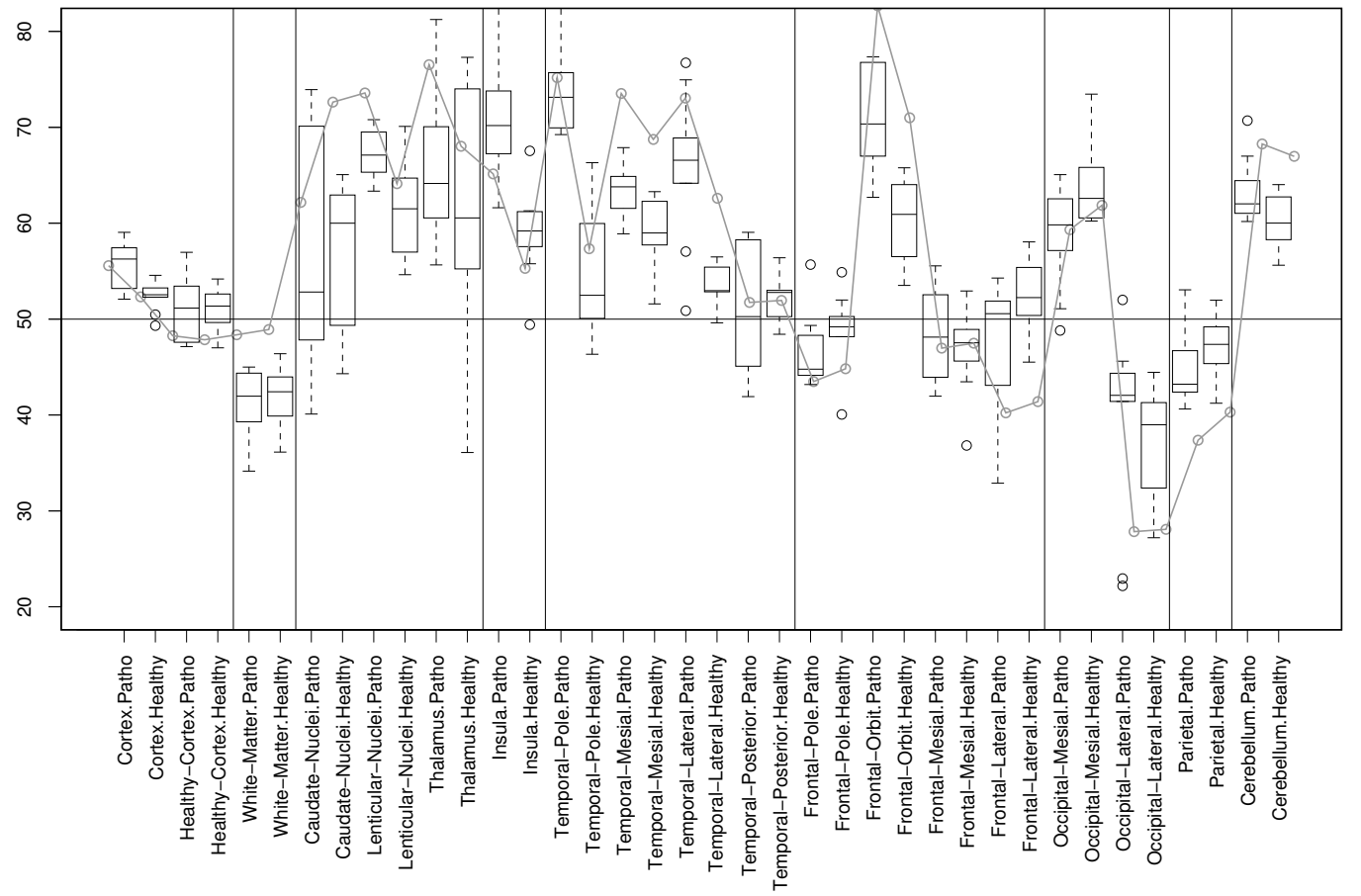

Figure 5. Distribution of AC-PVC perfusion measurements at each VOI from ictal SPECT of 10 MTLE patients ; The grey curve indicates AC-PVC perfusion measurements performed on the ictal simulated SPECT (straight line = activity of 50 counts/voxel)
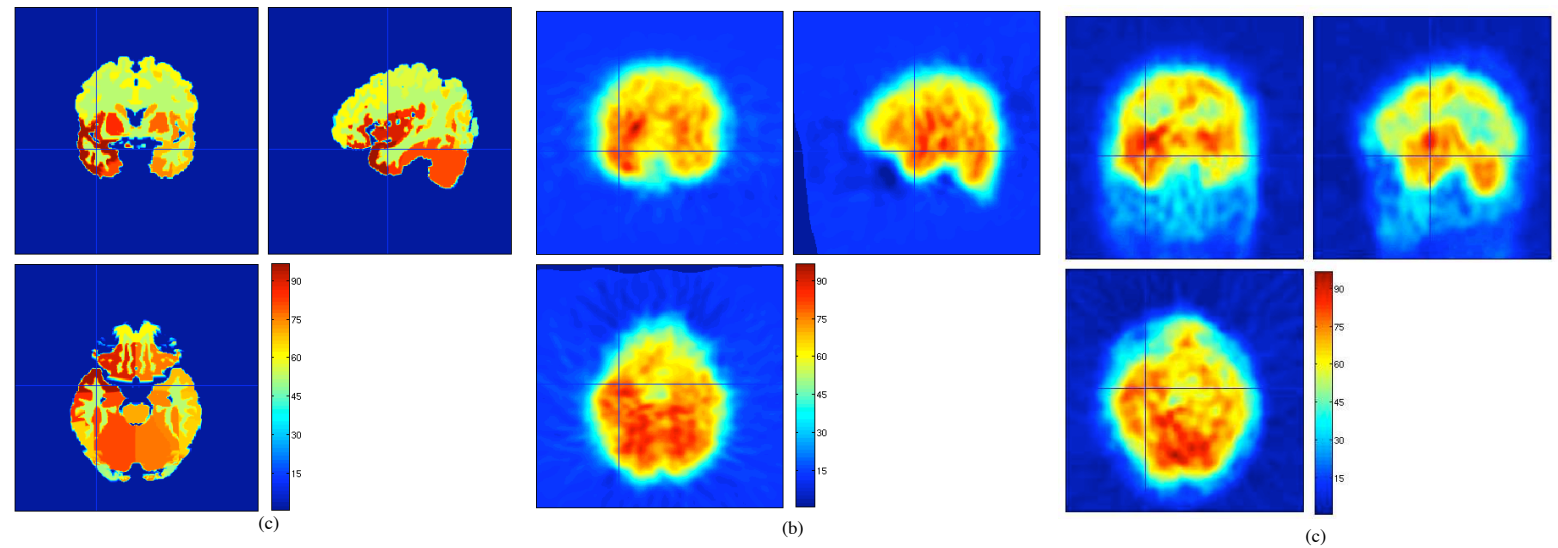

Figure 6. (a) Average theoretical model of ictal perfusion (MTLE) ; (b) Images reconstructed from the simulation of an ictal SPECT perfusion scan (MTLE) ; (c) Example of real ictal SPECT data from one MTLE patient (both real and simulated SPECT being only corrected for attenuation) 


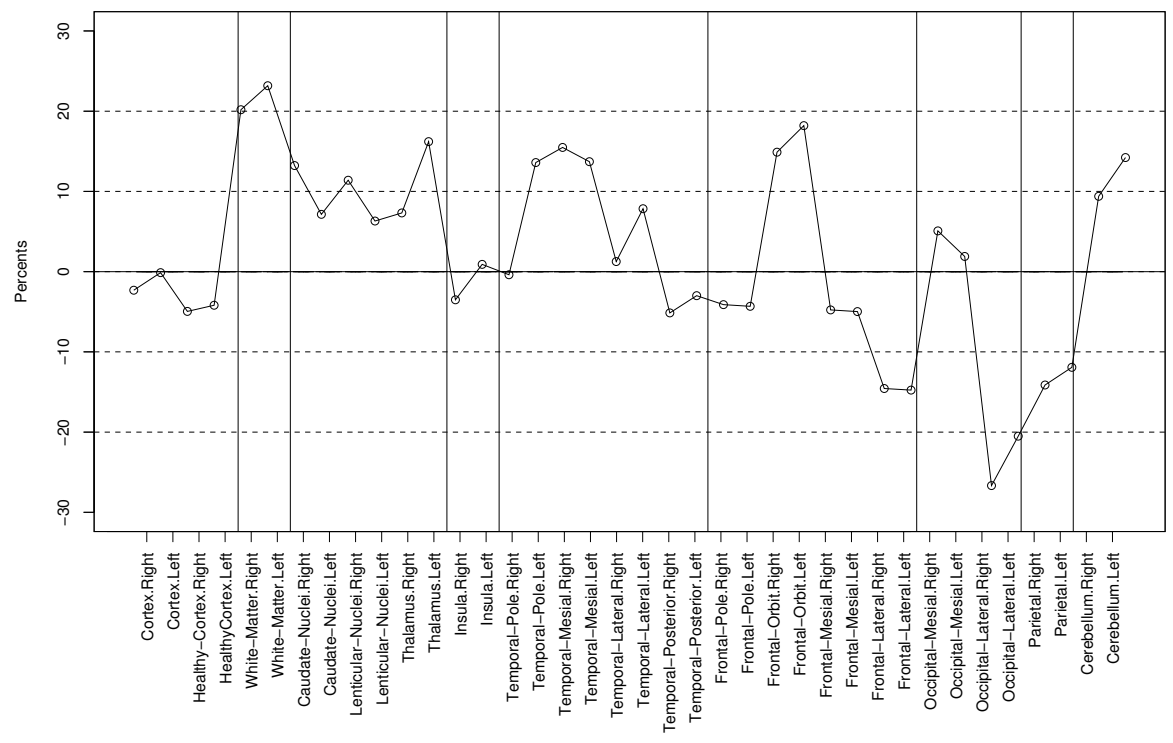

(a)

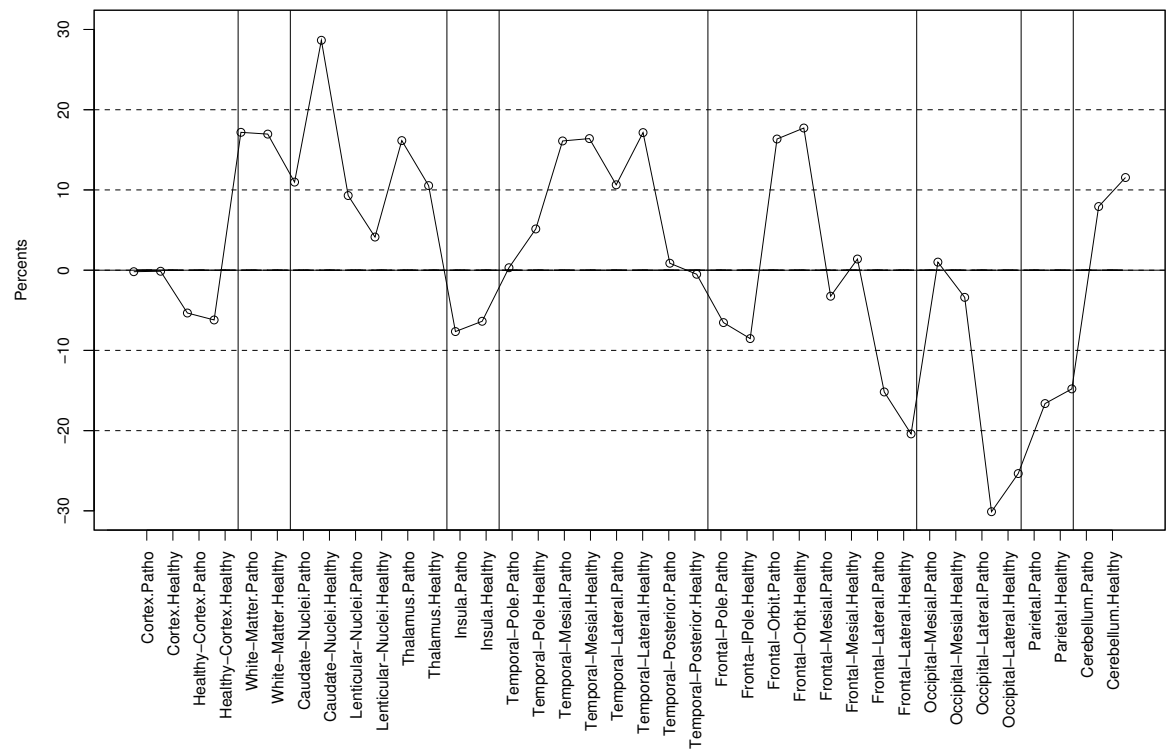

(b)

Figure 7. Relative assessment of the realism of normal and ictal simulated SPECT data: relative quantification differences $\operatorname{Diff} f_{i}=\left(\bar{s}_{i}-\bar{x}_{i}\right) / \bar{x}_{i}$ in $\%$, for each VOI $i$, taking into account as reference value the mean $\bar{x}_{i}$ perfusion measurement estimated on corresponding real SPECT data ; (a) Assessment of normal simulated SPECT based on SAC-PVC perfusion measurements performed on both real and simulated data ; (b) Assessment of ictal simulated SPECT based on AC-PVC perfusion measurements performed on both real and simulated data. 


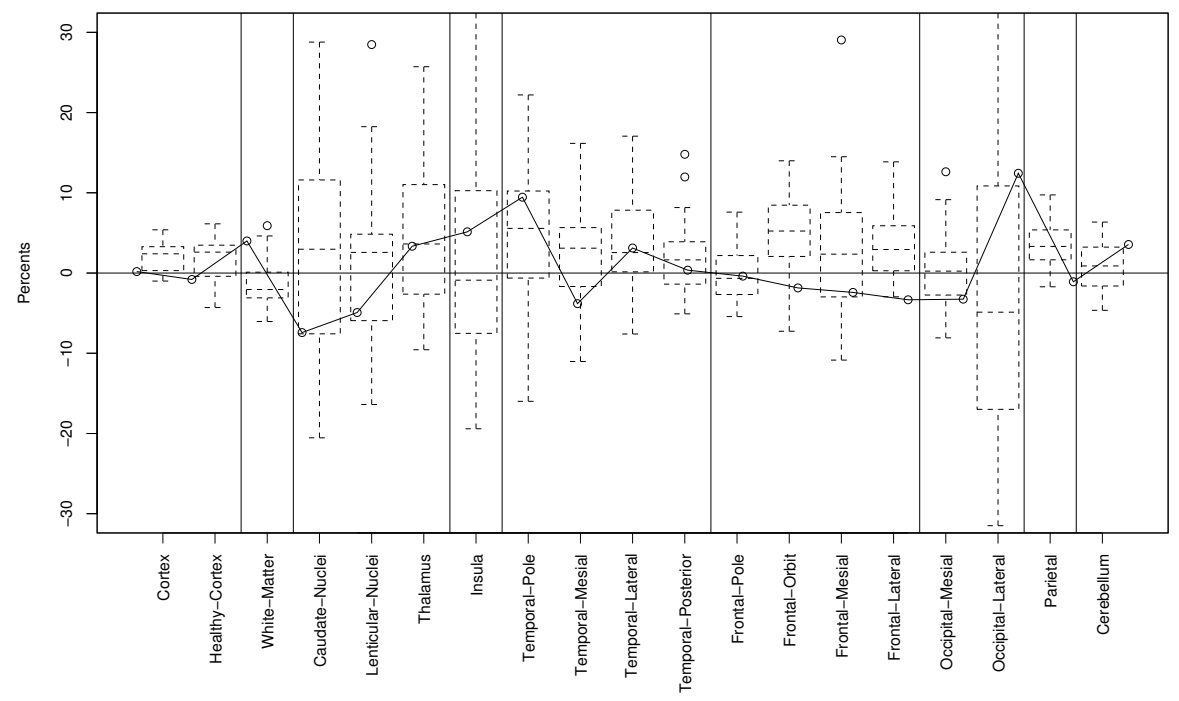

(a)

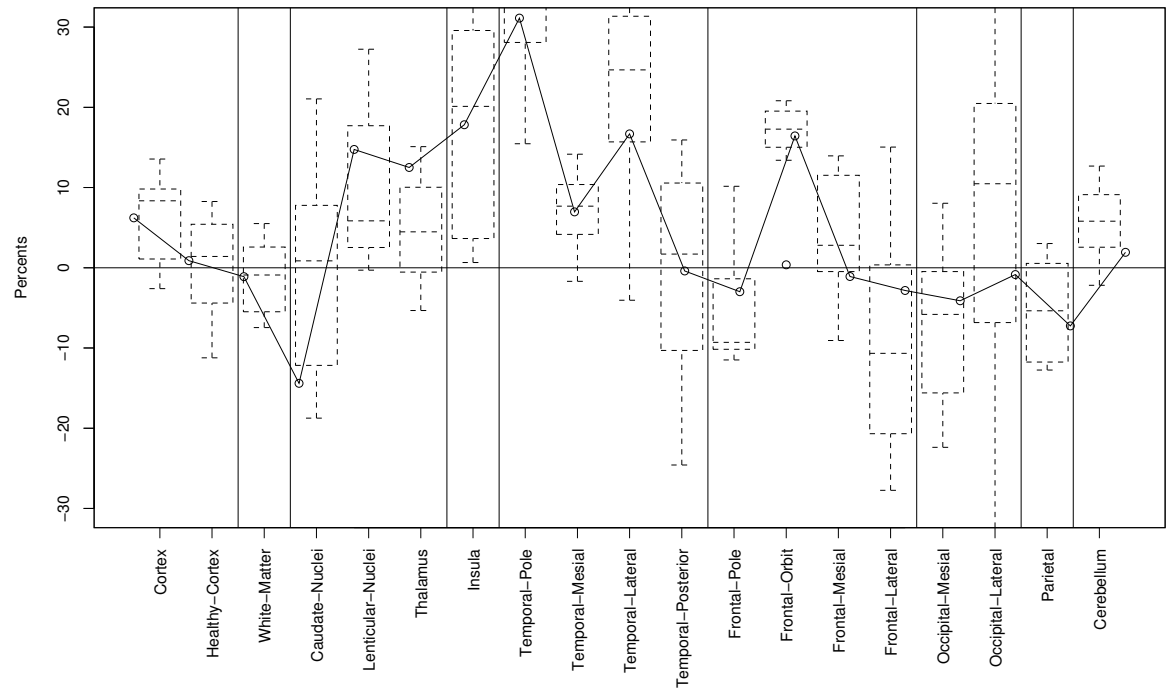

(b)

Figure 8. Inter-hemispheric asymmetry indexes measured on normal and ictal SPECT simulations: for normal SPECT simulations, inter-hemispheric relative differences $\left(\bar{s}_{i, \text { Diff }}=\left(\bar{s}_{i, L}-\bar{s}_{i, R}\right) / \bar{s}_{i, R}\right)$ in \% taking into account the right hemisphere $\bar{s}_{i, R}$ as a reference, and for ictal SPECT simulation, inter-hemispheric relative differences $\left(\bar{s}_{i, D i f f}=\left(\bar{s}_{i, P}-\bar{s}_{i, H}\right) / \bar{s}_{i, H}\right)$ in \% taking into account the healthy hemisphere $\bar{s}_{i, H}$ as a reference ; (a) Results for normal simulated SPECT (SAC-PVC perfusion measurements) ; (b) Results for ictal simulated SPECT (AC-PVC perfusion measurements) ; Distribution of inter-hemispheric asymmetry indexes measured on corresponding real data are shown using dashed boxplot. 